\title{
Discussion to the paper by O. P. Norlander and I. Nordén
}

Ghadiali. I would like to congratulate Dr Norlander not only on the presentation of his excellent paper but also for the fine work that is being done in his department. It is very difficult to find anything to disagree with or to criticize in what he has said. I have only a few comments to make which are based on our experience with a similar group of patients at Brompton Hospital. We have come more and more to appreciate the great desirability of isolating seriously ill patients from each other, not only from the point of view of cross-infection but also to protect the conscious patients from the mental trauma of the incessant noise and activity in a busy intensive care unit. In the management of our patients we rely on the arterio-venous oxygen difference and the urinary output as an index of the cardiac output over and above any other symptoms and signs. The monitoring of right and left atrial pressures separately helps us in assessing the myocardial state. We, like Dr Norlander, have no doubts whatever about the value of artificial ventilation and in fact any patient who is critically ill is put on the ventilator whatever the cause of his condition. We try to prevent at all costs the patient fighting the ventilator and therefore use curare in preference to large doses of opiates which may or may not have the desired effect and are potential depressants of the cardio-vascular system.

Ambiavagar. Dr Norlander's paper was most informative and interesting. He has drawn a number of conclusions, based on scientific measurement, that seem rather far-reaching on casual inspection. As I am unable to accept these conclusions at the moment, may I ask a few questions, the answers to which I am sure will help clarify the position.

Firstly, regarding the use of cardiac output measurements, Dr Ghadiali stated that if patients are very ill after cardio-thoracic surgery, respirator treatment was employed. Dr Norlander described measurements in similar circumstances that perhaps explain this clinical observation. How was the cardiac output measured Dr Norlander? And how was oxygen consumption on and off the ventilator, measured?

NoRlander. Cardiac output values were computed from oxygen consumption and arterio-venous oxygen differences according to the Fick principle. Oxygen consumption was measured with a special technique utilizing the EHN spirometer (Engström, Herzog \& Norlander, 1961) which allows measurement of oxygen consumption even in the presence of inert gases, e.g. nitrous oxide or other anaesthetic gases.

Ambiavagar. In the equation you have used for measuring pulmonary shunting, I presume the A-V difference referred to is the arterio-venous oxygen saturation difference. Could you indicate where the mixed venous sample was obtained from and what you consider to be the accuracy of your method? I ask because many of the values in column 1 of Fig. 4 are smaller than the generally accepted normal values which result from the admixture effect from bronchial veins and myocardial venous blood. Also, why does pulmonary shunting increase after valve repairs?

NORLANDER. The explanation of the method using $\mathrm{A}-\mathrm{V}$ differences and shunting are found in Gordh, Linderholm \& Norlander (1958) and Thung \& Norlander
(1966). Mixed venous blood was obtained from the body or inflow tract of the right atrium and $I$ agree that our method may not be too accurate on this point. However, as we measured the changes rather than the absolute values, I believe the results merit attention. The reasons for shunting, I believe, are several, such as positioning of the patient, mechanical atelectasis due to compression by fluid, gross alterations in perfusion-ventilation ratios due to changes in pulmonary blood-volume, etc.

Ambiavagar. Column 4 of Fig. 3 (with the patient on the respirator) shows that the patient had a cardiac output of $61 / \mathrm{min}$ and an oxygen uptake of $280 \mathrm{ml} / \mathrm{min}$. During spontaneous breathing the cardiac output increased to 8.7 and the oxygen uptake to $320 \mathrm{ml} / \mathrm{min}$. Later on-as shown in the last two columns-the oxygen consumption was 450 breathing spontaneously and the cardiac ouput was $7.2 \mathrm{l} / \mathrm{min}$. There was a $30 \%$ pulmonary shunt. On the respirator, the shunt fell somewhat - to $21 \%$ - and the cardiac output to 6.0 litres. There was, however, no significant change in oxygen consumption. Neither Figs. 3 nor 4 support your contention that artificial ventilation when substituted for spontaneous breathing reduced oxygen consumption by $13-50 \%$.

NORLANDER. The high oxygen consumption at the end of operation was certainly an additional sign that the patient needed the respirator. Besides that, already at that time it was clinically evident that the patient was on the borderline of her physical capacity. The next determination, approximately $15 \mathrm{hr}$ later, i.e. the next postoperative morning, the patient's condition was clinically satisfactory. However, even the short period off the respirator for the measurement rapidly led to deterioration. The relatively small change in oxygen uptake therefore may be interpreted as either due to insufficient time allowed during the determination for the uptake to increase, or to the fact that the patient was already on the borderline with maximum cardiac work, which could not be increased. Poor tissue perfusion would probably have been evident within a short time (total available oxygen decreases). This is an observation I have made in seriously ill patients at such a stage. This may not necessarily be associated with the muscular work of breathing but rather with the over-all malfunction of the cardio-pulmonary system. After all, the patient must sooner or later reach a stage where an increase in oxygen consumption is not possible. When this point arrives the patient usually rapidly develops cellular hypoxia with acidosis. At this point, respirator treatment is the only choice, if the patient is to stand any chance of survival.

Fig. 4, columns 2 and 5: At column 5 the patient was in no need of the respirator. The Figure supports the theory of respirator treatment beautifully: When the patient had an acute cardiac insufficiency due to breakdown of the aortic leaflet inserted at OP I, cardiac output increased to 8.9 litres. At the end of OP II, the patient had a high oxygen uptake and a small cardiac output, a syndrome occasionally seen after aortic valve surgery. In this type of patient any extra load on the respiratory system leads to a serious situation for the heart and may lead to death if active measures, such as respirator treatment, are not undertaken.

AmBiavagar. How were peak airway pressures and 
compliance pressures measured and how was this information used clinically?

NORLANDER. The peak dynamic pressure is observed on the manometer in the patient circuit of the Engström respirator, the compliance pressure at the end of the tidal volume, when the respirator maintains a 'plateau', i.e. no gas-flow except that delivered by the flowmeters, which can be neglected. Those two pressures are easily observed at the respirator. The changes in compliance provide useful information on the condition of the lung: a continuous decrease is usually a forerunner of pulmonary oedema or intrathoracic bleeding.

Ambiavagar. Lastly, how was intrapulmonary gas mixing studied?

NORLANDER. This was studied in dogs with artificially created strictures of the main bronchi and measured as pressure and carbon dioxide and alveolar-arterial oxygen gradients.

Ambiavagar. Just one last question. How was heart size measured?

NORLANDER. Heart size was measured with the patient in a supine position after a method described by Larsson \& Kjellberg (1948).

\section{Reference}

LARsSon, H. \& KJellberG, S.R. (1948) Roentgenological heart volume determination with regard to pulse rate and position of the body. Acta radiol. scand. 29, 159.

GHADIALI. In spite of precautionary measures the tracheostomies and the air passages of a number of patients become infected with Pseudomonas pyocyanea after a time. How best can prevention of tracheostomy cross-infection be ensured?

Williams. It seems to me that there is now clear evidence that antibiotic prophylaxis is only of use in a number of rather special situations, in particular where you are trying to prevent infection with a bacterium-such as the haemolytic streptococcus-that is known to be sensitive to a drug that can be used withough causing a profound alteration in the normal bacterial flora. 'Broad-spectrum' prophylaxis, aimed at preventing infection by some unspecified bacteria, has very rarely proved useful and has often proved dangerous. In experience with patients having renal transplantation we have found it far better to maintain a close bacteriological surveillance so as to detect infection at the earliest possible stage, when there is still time to determine the appropriate antibiotic before having to commence treatment. Probably the bacterial flora of the patient should be added to the list of characters that require to be monitored during intensive therapy.

Our own observations on bacteriological monitoring of the air in hospital wards show that this needs to be almost continuous if it is to be of use, for some of the 'broadcasts' of staphylococci into ward air are of very short duration.

The discussions in this Symposium have certainly shown that we need a great deal more information on the incidence and nature of infection in patients nursed in intensive care units. When staphylococcal infections are detected, the most likely sources of infection are other patients or members of the ward staff. Infections with Pseudomonas pyocyanea (aeruginosa), on the other $\stackrel{\mathbb{Q}}{\AA}$ hand, are much more likely to arise from contaminated $c$ humidifier units in ventilators and the like, or from contaminated disinfectant solutions. But, in addition, $\stackrel{\vec{D}}{+}$ evidence is now accumulating that patients become intestinal carriers of hospital strains of coliform organisms $\overline{\underline{\sigma}}$ and Pseudomonas in the same way as they acquire carriage $\frac{\bar{\omega}}{\sigma}$ of hospital staphylococci, so that the gut is a further $\overparen{\mathbb{D}}$ possible source of infection. Pseudomonas pyocyanea is the outstanding example of a bacterium that invades $\infty$ patients after broad-spectrum or intensive chemotherapy. $\vec{\circ}$

Crampton SMrTH. When we began to treat patients in the Respiration Unit at Oxford we made no attempt to $\vec{\omega}$ make aspiration of the trachea an aseptic proceeding. $\frac{\Omega}{8}$ We rationalized this omission by convincing ourselves ${ }_{0}$ that if we made aspiration too difficult a procedure, the 3 nurse would not aspirate the trachea often enough.

Some years ago we felt that this indifference to an $\omega$ obvious route for cross-infection could not continue $\overrightarrow{0}$ and we conducted a small study. We made a model of the is situation which was in fact a glass tube bent at something $V$ like the curve of the tracheotomy tube which, however, 0 was not joined to a trachea but to another glass tube containing a suitable culture medium. We held these $\vec{\longrightarrow}$ autoclaved models in the middle of a patient's bed and $\frac{7}{0}$ asked any nurse who passed by to go through the motions of aspirating this model as she would a patient's trachea. $\overrightarrow{0}$ We found that almost $100 \%$ of these models were contaminated with unpleasant pathogens.

We than scrubbed up, put on gown, mask, gloves, hat and repeated the experiment. We were not surprised to find that we did not in these circumstances contaminate any of the models. We then began to take off our protective clothing and we found that we could get down to one sterile glove on an unwashed hand and, with a little care to avoid touching the catheter with anything but the sterile glove, were still able to avoid contamination. This was further simplified by substituting a disposable unsterile glove for the sterile one. We have tested these disposable gloves on many occasions and although we have found organisms on them we have never found a pathogen. They have the advantage of being very much cheaper than the sterilized variety.

At the same time, however, we were investigating contamination of the tracheostomy wound, the tracheotomy tube and the bronchi. We took some pains to design a bronchial swab which was unlikely to be contaminated while it passed through the tracheotomy tube. On a significant number of occasions we found that a pathogen could be identified first in the tracheotomy wound, $24 \mathrm{hr}$ later in the tracheotomy tube, and $24 \mathrm{hr}$ after that could be identified in the bronchial tree. It therefore became obvious that one glove was not sufficient, and now one glove is used to prevent contamination of the aspirating catheter, and the other glove to prevent contamination of the tracheotomy wound, so two gloves are currently used in the Respiration Unit during aspiration of the trachea. The gloves which we use are about twopence each and it is quite easy to use 1000 gloves a week. We therefore find it necessary to be selective and decide in advance which patients shall be aspirated with gloves and which shall not. No patient, for instance, who has a vital capacity of over $500 \mathrm{ml}$ would qualify for gloves. We also spray the tracheostomy 
wound hourly with Polybactrin spray and apply Nystatin powder 8-hourly.

Williams. Dr Norlander referred to the need for staff working in intensive therapy units to wear special clothing. This may have disciplinary or psychological advantages but it is worth recalling the recent work of Noble, Shooter and Blowers (Shooter, 1966) on dispersal of staphylococci by carriers: the nature (and even the presence) of clothing has very little effect on the numbers shed. And Shooter's experiments (Speers et al., 1965) show that taking a shower bath actually increases the number of staphylococci that are dispersed from the skin. By all means let us have a uniform for the staff of the unit but let us be clear just what function it is supposed to perform.

\section{References}

SHOOTER, R.A. (1966) Infection in hospital. Abstr. Wld Med. $39,265$.

Speers, R., Bernard, H., O'Grady, F. \& Shooter, R.A. (1965) Increased disposal of skin bacteria into the air after shower baths. Lancet, i, 478.

QUESTION FROM THE FLOOR. What is the best method of sterilizing ventilators?

GHADIALI. At the Brompton Hospital a simple method of sterilization by ethylene oxide gas in a plastic bag is used. Stringent bacteriological testing has proved its value.

NORLANDER. In reply to the question on sterilization of ventilators, I mentioned in my paper that we have for a couple of months employed a new method for sterilizaion-or rather disinfection-of respirator equipment. The method is based on ultrasonic nebulization. We are using an ultrasonic aerosol of $70 \%$ alcohol in water in a closed circuit containing pure nitrogen. This method has the advantage that all internal parts of the respirator, for instance the rebreathing bag, valves, etc., may be properly disinfected. This method does not work when water is present and therefore the humidifier has to be bypassed during the procedure or has to be emptied and dried. We have taken bacteriological cultures before and after this procedure and although our results so far do not allow us to make a statistical analysis I may say that we are satisfied with this method. In addition to the usual mechanical way of cleaning the equipment, which I mentioned in my earlier presentation, this increased the scope for sterilization and cleaning of anaesthetic equipment. We never use a respirator before we have negative bacteriological cultures from at least three or four different sites on the machine.

KRISTENSEN: As pointed out, bacterial infections during prolonged respirator treatment in intensive care units represent a serious problem. In the group of patients from the Blegdamshospitalet presented here, infections were the most frequent cause of death during respirator treatment. Some precautions were taken to minimize the rate of infections in our patients. Care was taken to secure that all catheters introduced into the trachea for suctioning were sterile, but the nurses who performed the aspirations did not wear gloves. The hoses and the humidifier of the respirator were cleaned with soap and a solution of $3 \%$ phenol, after which no bacteria could be cultivated. This procedure was performed only when the respirator treatment of one patient had come to an end and the respirator had to be used for a new patient. No attempt was made to sterilize the respirator equipment during prolonged treatment of the same patient. Recently, as an experiment, various disinfectants have been added to the water in the humidifier.

Crampton Smith. Mr Chairman, may I make a plea that the word 'sophisticated' be expunged from the records of this symposium. I dropped into the Cohen Library on my way to this session and found that the shorter Oxford Dictionary defines 'sophisticated' under three headings:

(1) Mixed with some foreign substance: adulterated, not pure or genuine.

(2) Altered from or deprived of primitive simplicity or naturalness.

(3) Falsified in a greater or lesser degree.

The quotation is from Dryden, 'I love not a sophisticated truth with an alloy of lye in't'.

Although some of us who make these measurements may believe that they are sophisticated it seems a pity to define them as such! 